\title{
NEOPLASIAS ASSOCIADAS AO PÊNFIGO PARANEOPLÁSICO NO IDOSO: UMA REVISÃO SISTEMÁTICA
}

Bruna Tífani Bitzcof, Faculdade Meridional (IMED), brunaabitzcof@gmail.com Julia Quadri Bortoli, Faculdade Meridional (IMED), bortoli.jq@gmail.com Matheus Panosso Zanco, Faculdade Meridional (IMED), matheus_p_z@hotmail.com Vinícius Pasqual Montoya, Faculdade Meridional (IMED), vini-montoya@hotmail.com Alexandra Brugnera Nunes de Mattos, Médica Dermatologista Orientadora, anunes@hotmail.com

\section{RESUMO}

Introdução: O pênfigo paraneoplásico (PNP) é uma doença autoimune com apresentação cutânea bolhosa, associada ao desenvolvimento de tumores em órgãos internos. Por isso, a identificação precoce pode auxiliar no tratamento e sobrevida desses pacientes.Objetivo: Compreender o PNP como indicador tumoral e suas principais neoplasias associadas já relatadas em indivíduos com mais de 65 anos.Métodos: Realizou-se uma revisão sistemática utilizando as bases de dados PubMed, Scielo, Lilacs, BVS e Cochrane Library. Termos de pesquisa "paraneoplastic syndrome" AND "elderly" AND "pemphigus". Critérios de inclusão: artigos que abordam o PNP em indivíduos idosos, limitados entre 2013 a 2020. Critérios de exclusão: linguagem sem ser português, inglês ou espanhol; ser revisão sistemática; ou repetição.Resultados: Foram selecionados 30 artigos. A média de idade dos indivíduos acometidos com PNP é de aproximada de 73 anos. A neoplasia associada mais encontrada foi o linfoma folicular em 5 artigos. As lesões do pênfigo surgiram antes da neoplasia em 6 casos relatados. A completa remissão do pênfigo foi observada em 13 relatos, sendo sete desses advindos do tratamento da neoplasia associada após uma média de 3 meses.Conclusão: A remissão das lesões do PNP, bem como seu agravamento, está intimamente ligada à evolução do quadro tumoral. A fim de obter uma terapêutica direcionada a melhora das lesões e do bem-estar do indivíduo idoso, é importante estar atento ao diagnóstico precoce.

Palavras-chave: Síndrome paraneoplásica; Pênfigo Paraneoplásico; Idoso. 